\title{
Precise Localization and Formation Control of Swarm Robots via Wireless Sensor Networks
}

\author{
Han Wu, Shizhen Qu, Dongdong Xu, and Chunlin Chen \\ Department of Control and System Engineering, School of Management and Engineering, Nanjing University, \\ Nanjing 210093, China \\ Correspondence should be addressed to Dongdong Xu; xddnju@126.com
}

Received 10 August 2014; Accepted 20 October 2014; Published 11 November 2014

Academic Editor: Guangming Xie

Copyright (C) $2014 \mathrm{Han} \mathrm{Wu}$ et al. This is an open access article distributed under the Creative Commons Attribution License, which permits unrestricted use, distribution, and reproduction in any medium, provided the original work is properly cited.

\begin{abstract}
Precise localization and formation control are one of the key technologies to achieve coordination and control of swarm robots, which is also currently a bottleneck for practical applications of swarm robotic systems. Aiming at overcoming the limited individual perception and the difficulty of achieving precise localization and formation, a localization approach combining dead reckoning (DR) with wireless sensor network- (WSN-) based methods is proposed in this paper. Two kinds of WSN localization technologies are adopted in this paper, that is, ZigBee-based RSSI (received signal strength indication) global localization and electronic tag floors for calibration of local positioning. First, the DR localization information is combined with the ZigBee-based RSSI position information using the Kalman filter method to achieve precise global localization and maintain the robot formation. Then the electronic tag floors provide the robots with their precise coordinates in some local areas and enable the robot swarm to calibrate its formation by reducing the accumulated position errors. Hence, the overall performance of localization and formation control of the swarm robotic system is improved. Both of the simulation results and the experimental results on a real schematic system are given to demonstrate the success of the proposed approach.
\end{abstract}

\section{Introduction}

The application area of robot continues to expand since the 1980 s, and single robot can no longer satisfy the increasing requirement due to the complication of tasks and working environments. Since Beni developed several theories on cellular automata in the early eighties [1], self-organization multirobot system has drawn much attention of robot experts across the world because of its many advantages. IEEE International Conference on Robotics and Automation listed the robot coordination as a special subject in 1986. Several international conferences such as the Swarm Robotics and Swarm Intelligence have been held regularly in the last decade.

The swarm robotic system is a kind of special multirobot system which focuses the realization of individual physical robot as well as the interaction of robots with each other and the environment; that is, the related studies are concerned with how to design a relatively simple individual robot physically so that to get the desired collective behavior emerging from the interactions of robot to robot and robot to environment. Compared with the centralized control system, the structure of robots in a swarm robotic system which can fulfill the same task is relatively simpler. So the individuals in a swarm robotic system are interchangeable and easy to modularize and suitable for mass production. Generally, a swarm robot system is highly redundant. Therefore it has strong robust performance and the ability to adapt to the environment dynamically, which exceeds the capability of other kinds of robot systems, especially the single robot system. Swarm robotic systems are used in many occasions, such as organizing robots into certain formations, deployment of distributed sensors, mapping of the environment, and goal-searching $[1,2]$. Although the swarm robot system derives from the multirobot system, there are some major differences comparing with other multirobot systems: the robot's autonomy, large number of the robots, homogeneity of the robots, the incapability and inefficiency which can also be interpreted as simplicity of a single robot, and the limited sensing range [3]. 
Research on swarm robots has gradually emerged in recent years along with the development of multirobot systems. Fukuda is the first to attempt a collaborative way of working for a team of robots in his study of CEBOT [4]. CEBOT is a distributed system made of many indiscriminate autonomous robots which have limited intelligence on their own. The swarm intelligence emerges when the single robots interact with each other in the system they formed, in this way a complex task which outranges the capability of single robot can be fulfilled. The MARTHA (multiple autonomous robots system for transport and handling application) project specializes in a multirobot system, planning to set up a largescale team, made of 10 to 100 autonomous mobile robots, to carry cargoes in ports, airports, and other application scenarios [5]. Dorigo is the lead researcher of the SWARM-BOTS project sponsored by the Future and Emerging Technologies program of the European Commission [6]. They used sbots to conduct a series of experiments to achieve different goals. Seyfried et al. in Karlsruhe University of Germany have the I-SWARM project funded by the European Commission, intending to build the first very large-scale artificial swarm (VLSAS) with a swarm size of up to 1,000 microrobots with a planned size of $2 \times 2 \times 1 \mathrm{~mm}^{3}$ [7].

The further researches and applications of swarm robots depend much on the position information of the robot members in the system. There are many localization methods for mobile robots. Dead reckoning method is an effective and widely used localization method for mobile robots, which is usually utilized with other localization techniques to compensate its own defects $[8,9]$. Considering the requirements of low power and time consumption, data transfer speed, calculation complexity, and other characteristics of swarm robots, wireless sensor network is fit for the localization of swarm robot systems. Mao et al. give a general review of measurement techniques and localization algorithms in WSN, among which RSSI method proved to be suitable for multirobot localization [10]. Ishimoto and Hara use RSSI to calculate a set of common coordinates and heading direction for a robot swarm. Both indoor and outdoor environment experiment results are obtained and analyzed, though the data is not very satisfactory because of the poor ranging capability of RSSI [11]. Oliveira et al. proposed a relative localization algorithm for multirobot teams based on RSSI [12]. Although RSSI estimation is a coarse result, it is still sufficient for the robots to perform their tasks. So when the requirement of accuracy is not very high, RSSI can be used as an effective and efficient localization method [13, 14]. Zhang et al. proposed an environmental-adaptive path loss model to modify the traditional RSSI localization and obtained a desired result [15]. In the experiment of Dulimarta and Jain, mobile robots move from initial position to final position and avoid static or dynamic obstacles during navigation. They utilize the ceiling lights and door number plates as landmarks to complete the feedback and verification process of the robot's self-location, which makes a considerable improvement of localization accuracy in experimental results [16].

In this paper, we propose a hybrid localization method for swarm robots utilizing DR, WSN-based RSSI, and RFID techniques. Through the data fusion of Kalman filter and RFID-based calibration, the robot swarm is able to obtain its location with higher accuracy. The rest of the paper is organized as follows. In Section 2, the problem formulation, the model of a single robot, and the overall control structure are discussed. An improved localization method for swarm robots is proposed and analyzed in Section 3. Simulation results and schematic tests on real systems are given in Section 4 to verify the effectiveness and feasibility of the proposed approach. Conclusions are given in Section 5.

\section{Problem Formulation}

In this section, the problem of interest is first illustrated. Then a simplified model of an individual swarm robot is given and the formation control strategy is described.

2.1. Statement of the Problem. This paper focuses on the localization and formation control problem of a mobile swarm robotic system within a certain range of movement, using DR method and WSN to localize the robot and the electronic tag floors for position calibration. The robots work inside an office building and move through the corridors from an initial position to the goal position, maintaining the original formation during movement. Figure 1 gives a sketch of the designed scenario.

In most practical applications, the robot swarm should have a relatively accurate knowledge of each robot's position to perform its task efficiently. However, there are still defects in many localization methods for swarm robots and the lack of accuracy is the main problem. The localization method proposed in this paper aims at improving the precision of localization for swarm robots with no additional complexity in computation and hardware. Meanwhile an appropriate formation control strategy is adopted to keep the robots in a desired shape of formation.

2.2. Model of a Single Robot. A simplified model of a single robot in the robot swarm is described as shown in Figure 2 with the one step movement from step $k$ to step $k+1$. Consider the robot as a square with two driving wheels mounted on opposite sides across the central axis. The wheel gauge is the same as the robot's side length $l$.

The coordinate $x o y$ is defined as the world coordinate system. The robot's state vector $p$ at step $k$ is $p_{k}=$ $\left[x_{k}, y_{k}, \theta_{k}, v_{k}\right]^{T}$, where $\left(x_{k}, y_{k}\right)$ is the coordinate of the robot, $\theta_{k}$ is its orientation with respect to the $x$-axis of $x o y$, and $v_{k}$ is the robot's current velocity. $d_{k, l}$ and $d_{k, r}$ are the displacements of the left and right wheels from the $k$ th step to the $k+1$ th step, respectively. $d_{k}$ denotes the displacement of the robot's geometrical center, and $\Delta \theta_{k}$ denotes the robot's orientation change. Thus the $k+1$ th position can be obtained based on the position of $k$ th step. 


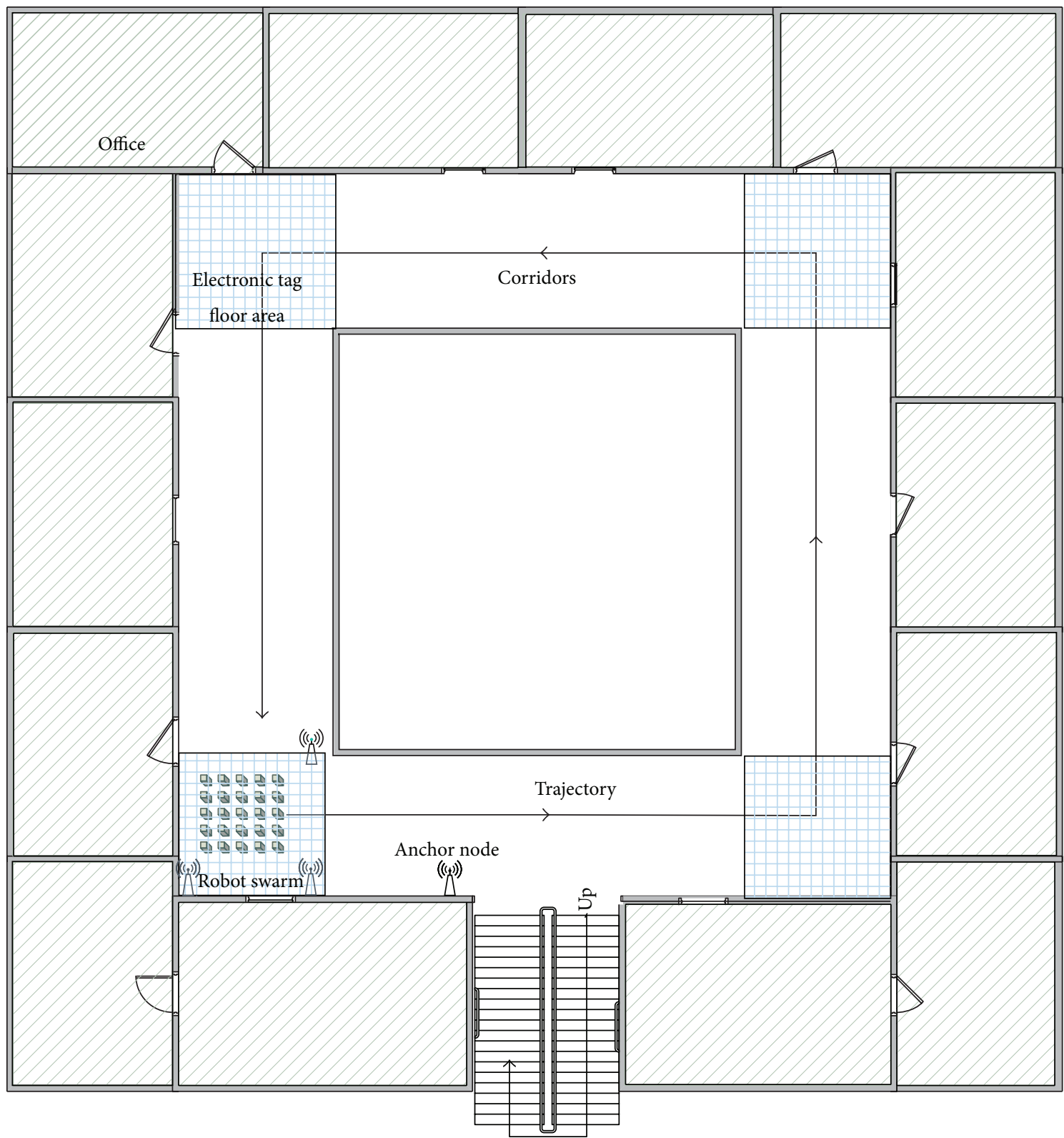

FIGURE 1: Sketch of the swarm robots moving in an office building environment.

The dead reckoning method gives an estimation of the robot's current position utilizing encoders. The process of calculating the robot's position by DR method can be described with the following equations:

$$
\begin{gathered}
d_{k}=\frac{d_{k, l}+d_{k, r}}{2}, \\
\Delta \theta_{k}=\frac{d_{k, r}-d_{k, l}}{l}, \\
\left(\begin{array}{l}
x_{k+1} \\
y_{k+1} \\
\theta_{k+1}
\end{array}\right)=\left(\begin{array}{c}
x_{k}+\Delta d_{k} \cos \left(\theta_{k}+\frac{\Delta \theta_{k}}{2}\right) \\
y_{k}+\Delta d_{k} \sin \left(\theta_{k}+\frac{\Delta \theta_{k}}{2}\right) \\
\theta_{k}+\Delta \theta_{k}
\end{array}\right) .
\end{gathered}
$$

The DR method only requires the previous coordinate and orientation; then the localization estimation of the next step can be obtained. This positioning method is efficient and has low requirements for hardware; thus it has been used widely for mobile robots. However, since the coordinate of next step is calculated on the basis of its previous step, the localization error accumulates through the whole motion process which leads to large deviation in a long distance due to the wheel slip, uneven ground, and many other errors. Generally there are systematic error and stochastic error for DR method. The systematic error mainly consists of the inequality of two wheels, the uncertainty of wheel gauge, the limitation of the encoder's resolution, and the limited sampling frequency. The stochastic error comes from wheel slipping and the difference of floor's roughness in different 


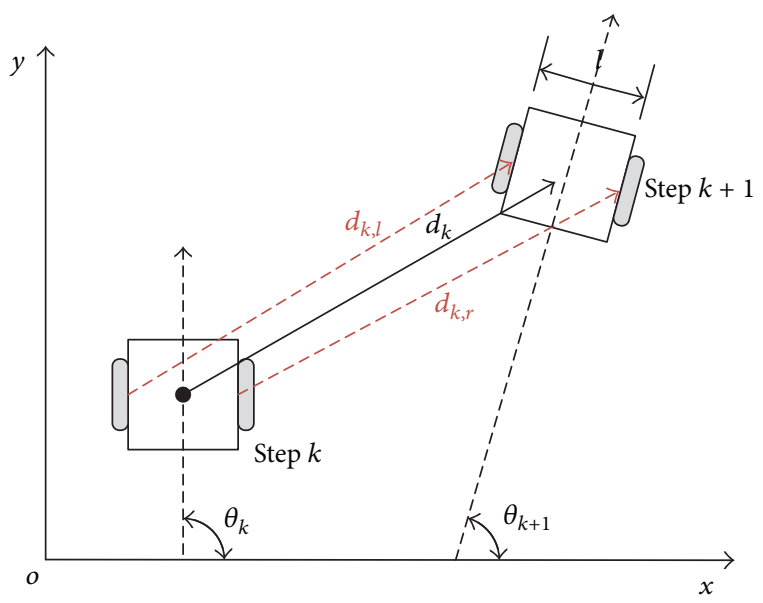

FigURE 2: Model of a single robot moving from step $k$ to step $k+1$.

areas. Since the stochastic error is unpredictable, a perfect solution to eliminate the error of dead reckoning is yet to be found. Due to the uncertainty of DR method, we have to use other kinds of sensors and localization methods to compensate.

2.3. Formation Control. In most application scenarios such as environment detection, reconnaissance, and surveillance, the robot swarm will be much more efficient and manageable when it is organized in a certain formation. There are several formation control strategies, such as behaviorbased approach combined with potential field [17], leaderfollower approach [18], and generalized coordinates and virtual structure methods [19]. Due to the simplicity of the single robot of swarm robots in equipment and low power consumption and other intrinsic properties, each robot only has the local information obtained by the limited sensors on board. Without centralized controller and a global knowledge of the rest robots' position, the formation of all the robots is organized through the communication and coordination with each other. Considering all the factors above, the leaderfollower strategy is most applicable in this scenario.

After forming a square with $7 \times 7$ robots in the starting position, the robot swarm continues to maintain the formation during the whole movement process. $p_{i, j}^{k}$ denotes the position of the robot in the $i$ th column and $j$ th row at the $k$ th sampling step. Figure 3 shows the formation of the robot swarm in the world coordinate system. Along the heading direction, the head of every column is assigned as the group leader and the robot at the far left in the front line is presumed as the global leader. Role assignment will change following the above rules when the swarm's heading direction changes. Each robot is considered as capable of knowing the distance and the difference of heading direction between each other. At each sampling step, every robot in the swarm detects its surroundings and decides the actions it will perform to correct the current formation. Global leader remains its position unchanged; group leaders adjust themselves in a straight line which is perpendicular to the heading direction

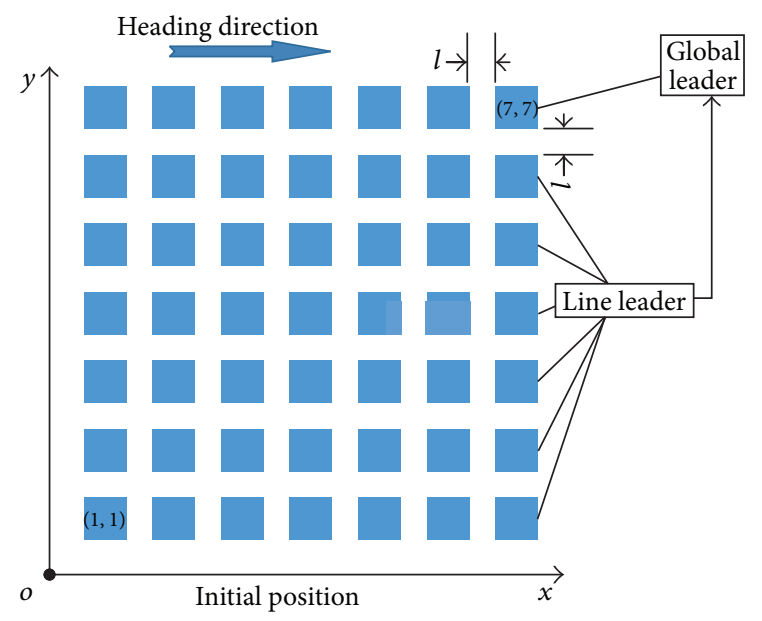

FIGURE 3: The square shape formation of the robot swarm in the initial position.

with the global leader and the followers in each group move in line with their group leaders along the heading direction.

\section{Localization and Formation Control with Wireless Sensor Networks}

Two kinds of WSN-based localization methods are utilized to help improve the performance of DR-based method, that is, RSSI-based localization method using ZigBee WSN and position calibration using electronic tag floors. In this work the designed scenario is that a robot swarm which has been organized into a certain shape of formation in the initial position moves to a target position while maintaining the original formation during the whole moving process. The beacon nodes of ZigBee WSN system and electronic tag floors are laid in some relevant locations within the movement range of the robot swarm. When a robot moves outside the areas covered by electronic floors, its position is calculated by DR and RSSI methods; once it reaches the areas covered by electronic tag floors, the robot obtains its coordinates from the detected electronic tag floor and eliminates the accumulated errors of DR- and RSSI-based localization method. In the designed scenario, the electronic tag floors are laid in the corners and thus can effectively eliminate the turning errors. The formation of the swarm robots is maintained by utilizing the leader-follower method. The flowchart of the designed approach is shown in Figure 4.

3.1. Overall Control Structure. The overall control structure is designed based on the properties of the swarm robots. Considering the large number of individual robots and their limited sensing range, centralized control strategy is not feasible for a swarm robot system. While distributed control fits for the requirements such as flexibility, reliability, openness, and easy maintenance, it has disadvantages like low efficiency. Thus we use a linear dominance hierarchy [20] as the overall control structure to achieve a balanced 


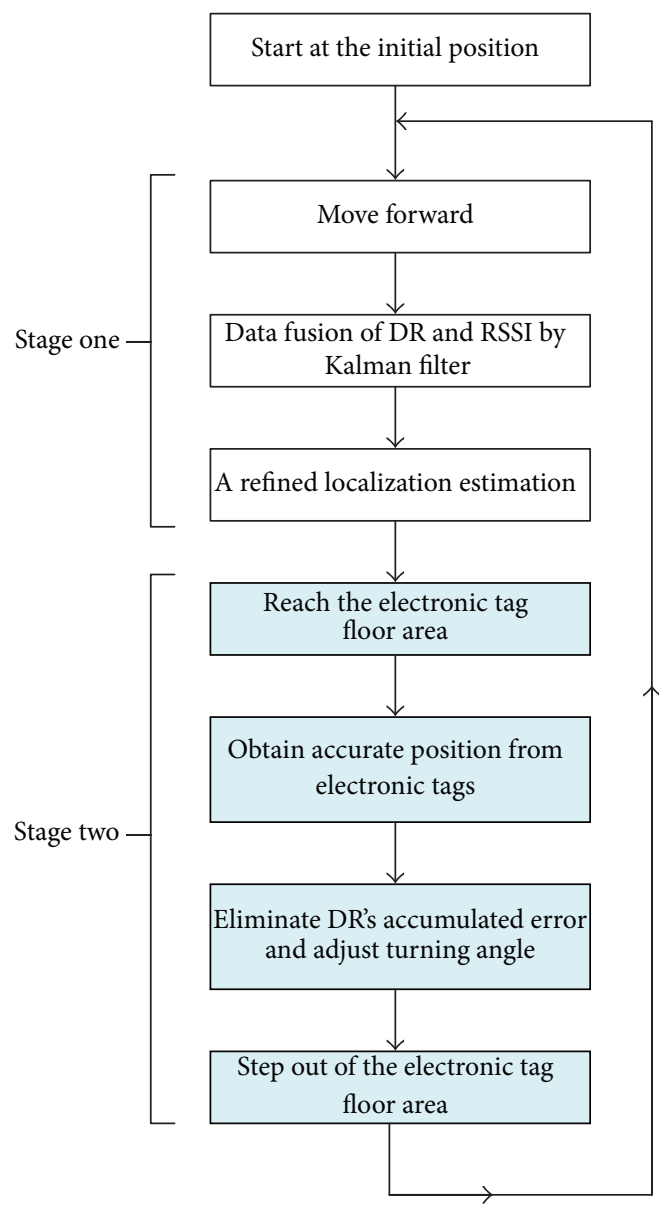

FIGURE 4: Flowchart of the designed approach.

performance in formation control of the whole swarm robotic system.

As shown in Figure 5, the control architecture is a distributed control system organized in a two-level linear hierarchy. Facing the robot swarm's heading direction, the leftmost robot in the front line is assigned as the global leader automatically. The rest robots in the front line work as the group leader, and the ones after them are the followers which comply with the leader-follower formation control strategy. It is assumed that the robots are equipped with infrared sensor that allows them to know the deviation of distance and direction between each other within a certain range, which is also used to avoid collisions of robots.

\subsection{Fusion of DR and RSSI}

3.2.1. ZigBee-Based RSSI Localization. The communication between swarm robots is a many-many process and it is achieved by the local area communication network. For reasons of economy and efficiency, requirements of transfer speed, distance, network scale, and power consumption of a robot swarm, ZigBee-based WSN is adopted in this paper for the communication between robots, and accordingly it is very convenient to implement the localization of each of the nodes. Generally speaking, there are three kinds of ranging methods in WSN localization, which are based on the angle-of-arrival, the distance estimation, and the RSS profiling measurements [10], respectively. As a kind of distance measurement technique, RSSI requires no more than the basic sensors and has low power consumption, which fits for the localization of small-size wireless mobile devices. In this paper, ZigBee-based RSSI localization method is applied to improve the DR method.

Each robot is equipped with the same ZigBee communication module, and it is assumed that the robot swarm has built up a wireless sensor network within its working environment. All the robots in the WSN can be positioned by the RSSI information. Based on many previous researches and experiments $[13,14]$, the received power $P(d)$ has a logarithmic relationship with the distance $d$ between the sender and the receiver, which can be described by the following equation:

$$
P(d)=P_{0}-10 n_{p} \log \frac{d}{d_{0}},
$$

where $P_{0}$ is the received power at the reference distance of $d_{0}, d$ is the distance between the sender and the receiver, and $n_{p}$ is the path-loss exponent. The value of $n_{p}$ can be determined by doing a series of empirical measurements of $P$ and $d$. Then with the knowledge of $P$, the value of $d$ can be obtained. Theoretically, with three reference nodes the robot (a blind node) is able to calculate its own position $p_{k \text {, RSSI }}$. With more reference nodes the localization estimation can achieve higher accuracy, but it also leads to higher energy consumption and computational complexity.

In fact, RSSI is an unstable indicator that suffers from a number of related parameter variations which can be divided into two kinds of influence factors: the propagation environment and the hardware devices. Environmental factors include the background noise, multipath propagation, the temperature, and obstacles within the propagation space. Hardware factors consist of the sending power, antenna's type and gains, the signal-noise ratio and supply voltage, and so forth [21]. Since the RSSI method is a quite coarse localization technique, it is only used as a complementary tool considering the topology of the whole localization system.

3.2.2. Kalman Filter. Kalman filtering, proposed in 1960 by R. E. Kalman, is a popular technique in multisensor data fusion, which includes two steps, prediction and calibration. Kalman filtering has been applied in robot localization, map building, navigation, object identification, and many other related fields for many years [22-27].

Consider the moving robot swarm as a discrete-time system which can be described as follows:

$$
\begin{gathered}
x_{k}=A x_{k-1}+B U_{k}+w_{k}, \\
z_{k}=H x_{k}+v_{k},
\end{gathered}
$$

where $x_{k}$ and $z_{k}$ are the actual and measured values at sampling step $k$, respectively; $A$ is the system matrix; $B$ is 


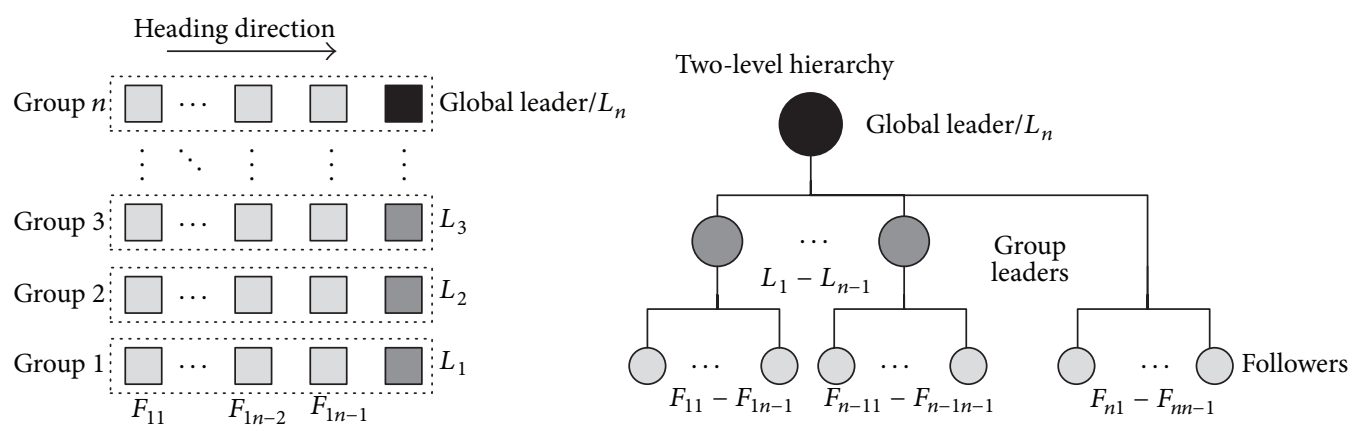

FIGURE 5: Overall control structure of the swarm robotic system.

the input matrix; $w_{k}$ is process covariance; $v_{k}$ is measurement covariance.

Assumed as white Gaussian noise, $w_{k}$ and $v_{k}$ follow normal distribution described in (4), where their covariances are denoted by $Q$ and $R$, respectively:

$$
\begin{gathered}
P_{w} \sim N\left(\mu_{w}, Q\right), \\
P_{v} \sim N\left(\mu_{v}, R\right) .
\end{gathered}
$$

Then the system state can be reconstructed utilizing Kalman filter as follows:

$$
\begin{gathered}
x_{k \mid k-1}=A x_{k-1 \mid k-1}+B U_{k}, \\
P_{k \mid k-1}=A P_{k-1 \mid k-1} A^{T}+Q, \\
\mathrm{KG}_{k}=\frac{P_{k \mid k-1} H^{T}}{\left(H P_{k \mid k-1} H^{T}+R\right)}, \\
x_{k \mid k}=x_{k \mid k-1}+\mathrm{KG}\left(z_{k}-H x_{k \mid k-1}\right), \\
P_{k \mid k}=\left(I-\mathrm{KG}_{k} H\right) P_{k \mid k-1},
\end{gathered}
$$

where $x_{k \mid k-1}$ and $x_{k \mid k}$ are the prior and posterior estimation of system state at step $k$, respectively, $P_{k \mid k-1}$ and $P_{k \mid k}$ are their covariances, $\mathrm{KG}_{k}$ is the optimal Kalman gain, and $I$ is the identity matrix. Based on (5), the minimum estimation error covariance can be acquired in time, described as

$$
P_{k \mid k}=E\left(e_{k \mid k} e_{k \mid k}^{T}\right)
$$

where $e_{k \mid k}=x_{k}-x_{k \mid k}, x_{k}$ is the actual state value [26].

The essence of Kalman filtering is to refactor system state vectors. Follow the prediction-measurement-calibration sequence and eliminate the random disturbance using the actual measurements; then the system state can be reconstructed.

3.2.3. Data Fusion by Kalman Filter. The movement process of the robot swarm can be divided into two stages, as shown in Figure 4. The first stage is moving forward in a straight line. The second stage starts when the robot's position estimation gets in the electronic tag floor areas, which triggers the RFID card reader mounted on the robot, and it ends when robot moves away from these areas.
During the first stage, the robots move forward, selfpositioning with Kalman filter by merging the data of DR and RSSI measurements. In DR method, systematic errors like the inequality of wheel diameter cannot be avoided but can be compensated by modeling the errors, while stochastic errors like wheel slipping can be treated as white Gaussian noise [25] and can be reduced by using Kalman filter. According to [28], the errors of $x, y$ coordinates and turning angle $\theta$ in DR-based localization estimation can be modeled as $\Delta x \sim N\left(\mu_{x}, \sigma_{x}^{2}\right)$, $\Delta y \sim N\left(\mu_{y}, \sigma_{y}^{2}\right)$, and $\Delta \theta \sim N\left(\mu_{\theta}, \sigma_{\theta}^{2}\right)$, respectively.

DR is used to get the prior estimation and RSSI estimation is used as the measured values. Then (5) can be adapted to a simpler version as follows:

$$
\begin{gathered}
\mathrm{KG}_{2, k}=\frac{\sigma_{1, k}^{2}}{\sigma_{1, k}^{2}+\sigma_{z_{2}, k}^{2}}, \\
\widehat{x}_{2, k}=\widehat{x}_{1, k}+\mathrm{KG}_{2}\left(Z_{2, k}-\widehat{x}_{1, k}\right),
\end{gathered}
$$

where $\mathrm{KG}_{2, k}$ is the Kalman gain used in data fusion and $\sigma_{1, \mathrm{k}}$ and $\sigma_{z_{2}, k}$ are the standard deviations of DR and RSSI estimation, which can be obtained statistically from experimental results. $\hat{x}_{1, k}$ and $\hat{x}_{2, k}$ are the prior and posterior estimation at step $k ; z_{2, k}$ is the RSSI measurements. Thus an optimized localization result can be obtained at each step by (7).

3.3. Calibration Using Electronic Tag. Radio frequency identification, also known as electronic tag, is used to identify a certain object and transfer data via radio signal with no need for mechanical or optical contact between identification system and target objects. The basic components of a RFID system are a tag, a reader, and an antenna. A tag has its exclusive electronic code and it is attached to the target object. A reader is a handheld or stationary device used to read or sometimes write data in the tag. An antenna completes the radiofrequency signal transmission tasks.

There are active tags, passive tags, and semipassive tags. Active tags have built-in batteries and emit signals periodically. Battery-assisted passive tags also have small batteries, which can be triggered only when the reader is nearby. Passive tags get their power supply from the radio waves of the reader instead of batteries. The data exchange will not happen until the reader comes close to the tag to power it up [29]. The default of battery makes passive tags cheaper and smaller, and 
the electronic floor we use in our work is a kind of passive tag. The RFID technology has been widely used and has plenty of potential applications in the future [30]. In this paper we use it in the localization system as fixed-point marks to calibrate the estimated position of swarm robots.

In the process of moving around, the robots will get into the areas where the electronic tag floors are previously distributed. For the sake of energy conservation, the RFID reader will be turned on only when the estimated position of the robots are close to the electronic tag floor area within a range of $0.5 \mathrm{~m}$. The ID of each electronic tag corresponds to a certain set of fixed coordinates, which was manually preset. Then the robot can obtain its actual location by any detected ID. Since the electronic tags are only floored in the topologically important areas, in this case, the turning corners, the robot can adjust its turning angle to the desirable orientation by continually collecting the errorless position information from the tags along its path. The localization method in stage one is suspended until the robots can no longer detect electronic tags and step out of the electronic tag floor areas. Once the robot leaves these areas, it steps into the stage one again. In this way the cycle repeats until the robot swarm arrives to its final destination.

An illustration of the calibration process of a single robot is shown in Figure 6. When the robot $R$ steps onto the floors at sampling step $m$, it obtains the first detected RFID signal, which can be transformed into its present coordinates. During the movement between two electronic tag floor areas, the uneven ground and the mechanical errors deflect $R$ from its ideal trajectory. In order to make a turn at the desired position, robot $R$ calculates the difference between its current position and the preset turning point denoted by the blue shaded floor and adjusts its heading direction. As it moves towards the turning point, $R$ keeps detecting IDs and uses them to navigate until it reaches the blue floor. Then $R$ turns a preset amount of angle, which inevitably has some mechanical errors. Robot $R$ navigates itself to the right heading direction and continues its following journey after stepping out of the electronic tag floors.

For the whole swarm, robots still comply with the leaderfollower method to maintain the formation. Once the global leader detected the first ID of an electronic tag, obtaining its precise location, then all the other robots correct their orientation and position based on their assigned roles. So far the localization errors are already eliminated to relatively very small values. When all the swarm members are inside the electronic tag floor area, the localization errors can be almost eliminated completely.

\section{Experimental Results}

4.1. Simulated Results. To verify the effectiveness of the proposed approach, a series of simulations are carried out using Matlab. All the related parameters are chosen based on real robot systems that are tested in an indoor office environment.

As shown in Figure 7, it can be seen that generally the deviation of DR method's estimation from the ideal position

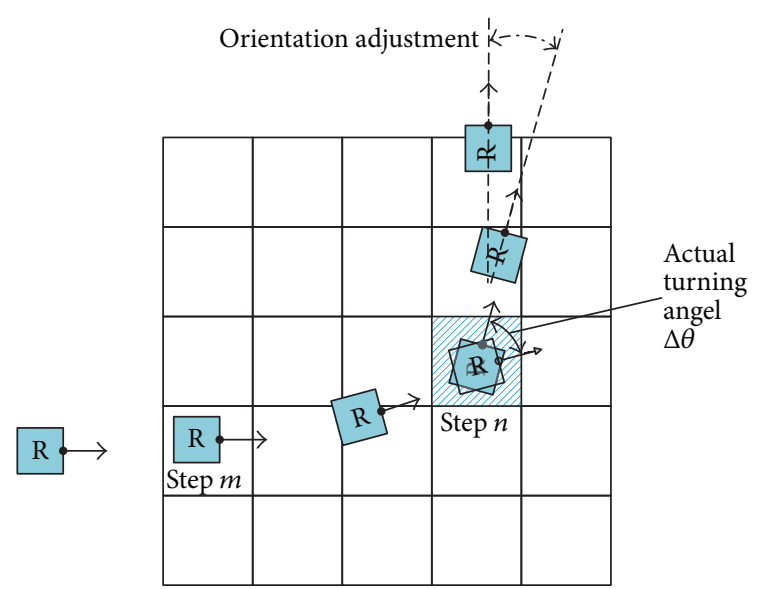

FIgURE 6: Position calibration for robots using electronic tag floors.

is quite large. As the movement distance increases, the errors accumulate to a great extent, which can be seen in the figure that the robot's trajectory is diverging during the movement from one corner to another. However, the trajectory converges when it reaches the turning corner covered by electronic tag floors, because the robot can obtain their precise location with an RFID reader receiving data from the electronic tags. So, as the robots move through the electronic tag floor area, they can adjust themselves to the right direction based on the coordinate information from the tags and thus there is no turning error. In this way, the robot starts its second, third, and fourth segment of path with no previous error. On the other hand, the deviation of the RSSI localization results varies in a certain range and does not accumulate as robots move forward. The localization results of the proposed approach demonstrate better performance and the deviation of the localization results is relatively small in general. All these simulation results show that the estimated coordinates of RSSI method are less accurate than DR method in a short distance, while, after a period of time, the error of DR method accumulates quickly and then becomes bigger than that of the RSSI method. By combining these two methods, the proposed approach has the least deviation. In addition, the electronic tag floors further improve the localization results by calibrating all the positions of robots that step into the electronic tag areas, which is very important for formation control of swarm robots.

Figures $8(\mathrm{a})$ and $8(\mathrm{~b})$ demonstrate the partial magnifications of the trajectories. From step $k=1$ to $k=8$, the deviation of DR estimation is relatively small while the RSSI estimation is far from the real trajectory, and the estimation of the proposed method is close to DR estimation. From step $k=8$ to $k=16$, DR's estimation gradually moves farther and farther away from the real trajectory; during this period, the estimation of the proposed method still keeps close to the real trajectory due to the balancing of RSSI results. Figure 8(c) shows the situation when a robot reaches the electronic tag floor area, where all the localization errors have been eliminated and the estimation matches perfectly the real position. As the robots move out of the corner area, localization errors begin to accumulate again. 


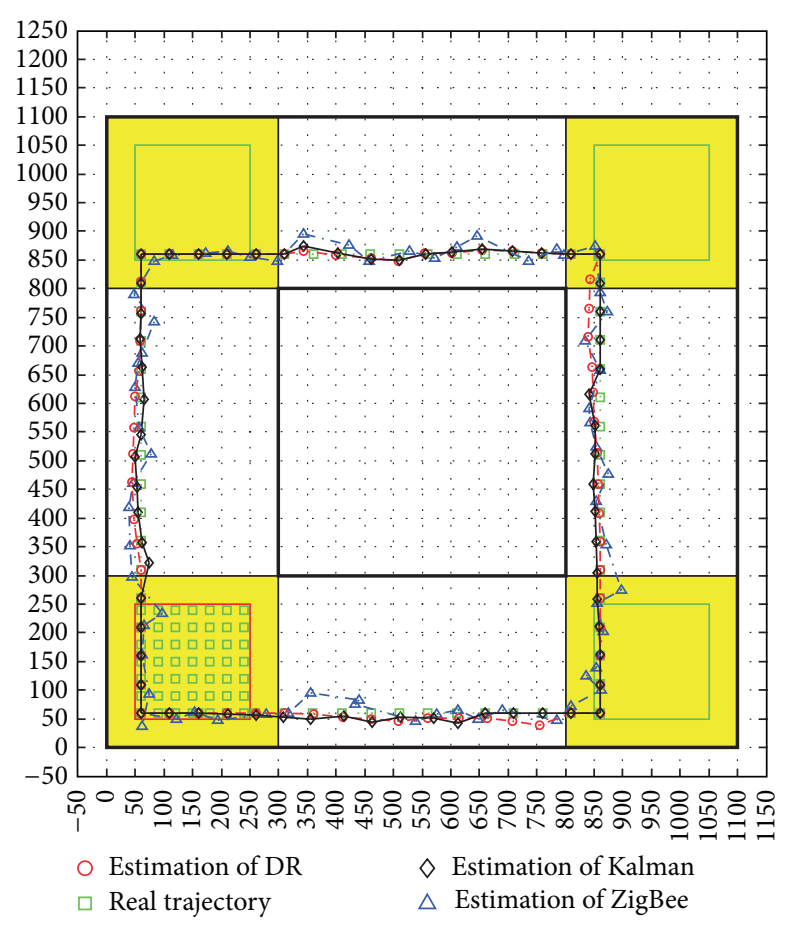

Figure 7: Estimated trajectories of all the three methods for the swarm robotic systems.

The deviation of each method is shown in Figure 9. There are four segments of the robot's trajectory and within each segment the red curve of DR is on the rise and then converges to zero when the robot gets to the electronic tag floor areas. The blue curve of RSSI deviation fluctuates and has no tendency to grow over time. The black curve of the proposed method's deviation is a balanced result between the DR method and the RSSI method, which is lower than both of DR and RSSI methods regarding the whole process of movement. The bars of three different colors on the right denote the mean error of all the robots in each step of DR, RSSI, and the proposed approach, respectively. It is clear that the proposed method has the least deviation.

Since the whole robot swarm corrects its formation before it moves towards the next step, the localization results of DR and the proposed method are influenced. Figure 10 shows the comparison on estimation errors of DR method before and after formation correction. From the perspective of the whole swarm, the localization errors of each step are smaller after the formation adjustment. The estimation results of the RSSI method are not affected by the formation control process, so the accuracy of its localization will not change and thus has no influence on the result of the proposed method. Figure 11 shows the comparison on estimation error of the proposed method before and after formation correction. The curve of the error after formation adjustment is not always below the one before formation adjustment, but the bar denoting the mean error of each step shows that the estimation error does decrease after adjusting the formation. Though the formation control may lead to an increase of the estimation error in some steps, there is a slightly drop of $0.4 \mathrm{~cm}$ on average.
TABLE 1: Total errors of the three methods.

\begin{tabular}{lccccc}
\hline$/ \mathrm{cm}$ & RSSI & \multicolumn{2}{c}{ Dead reckoning } & \multicolumn{2}{c}{ Proposed method } \\
\hline \multirow{3}{*}{$\sum_{k=1}^{n} S_{k}$} & 1334.90 & 408.42 & 377.97 & 360.75 & 335.28 \\
& 1350.22 & 395.39 & 362.07 & 349.26 & 392.92 \\
& 1333.80 & 385.98 & 354.06 & 339.87 & 307.89 \\
& 1349.20 & 386.36 & 355.66 & 344.04 & 194.80 \\
\hline Mean values & 1342.03 & 394.04 & 362.44 & 348.48 & 307.72 \\
\hline
\end{tabular}

TABLE 2: Average errors of the three methods at each step.

\begin{tabular}{lccccc}
\hline$/ \mathrm{cm}$ & RSSI & \multicolumn{2}{c}{ Dead reckoning } & \multicolumn{2}{c}{ Proposed method } \\
\hline \multirow{2}{*}{$\sum_{k=1}^{n} S_{k}$} & 20.55 & 6.28 & 5.81 & 5.55 & 5.16 \\
${ } }$ & 20.77 & 6.08 & 5.57 & 5.37 & 6.04 \\
& 20.52 & 5.94 & 5.45 & 5.23 & 4.74 \\
& 20.76 & 5.94 & 5.47 & 5.29 & 3.00 \\
\hline Mean values & 20.65 & 6.06 & 5.58 & 5.36 & 4.73 \\
\hline
\end{tabular}

Define the error $S_{k}$ as the deviation between estimation results and the real position at the $k$ th step:

$$
S_{k}=\sqrt{\left(x_{k}-x_{k}^{\prime}\right)^{2}+\left(y_{k}-y_{k}^{\prime}\right)^{2}},
$$

where $\left(x_{k}, y_{k}\right)$ is the real coordinates and the $\left(x_{k}^{\prime}, y_{k}^{\prime}\right)$ is the estimated coordinates.

Table 1 gives the total estimation errors of the three methods through the whole trajectory, where the numbers in italic are the results after formation adjustment. According to the leader-follower formation control strategy, the mean error of the whole swarm at each step is actually the value of the global leader, which is presented in Table 2. Based on the seven simulation tests, it can be seen from Table 3 that the errors tend to decrease after adjusting the formation. Theoretically the formation control does not affect the localization results of the whole swarm. However, in the proposed algorithm, group leaders and followers update their current locations with the values calculated by the leaderfollower rule. The errors in this location information only contain mechanical errors, which are definitely smaller than the errors of localization results estimated by any positioning method.

When the robot swarm moves more than one round along the hallway, the result remains the same as the first round since the electronic tag floors eliminate the accumulated error of DR method at each turn. Figure 12 gives an evident illustration during the second round, and the following more rounds are still the same situation as before.

4.2. Experimental Results on Real Systems. Experiments on real systems are carried out inside an $8 \times 8 \mathrm{~m}^{2}$ office room and four identical differential-drive mobile robots are used as a simplified robot swarm for schematic demonstration. A ZigBee WSN is built with 8 anchor nodes and 4 blind nodes mounted on the robots. The electronic tag floors are laid in the corners of a $5 \times 5 \mathrm{~m}^{2}$ square. There are $3 \times 3$ pieces of tagged floors in each corner, and each floor contains 9 evenly 


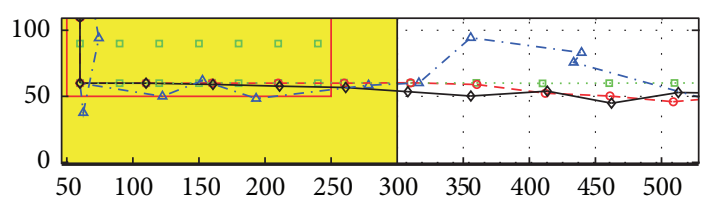

(a)

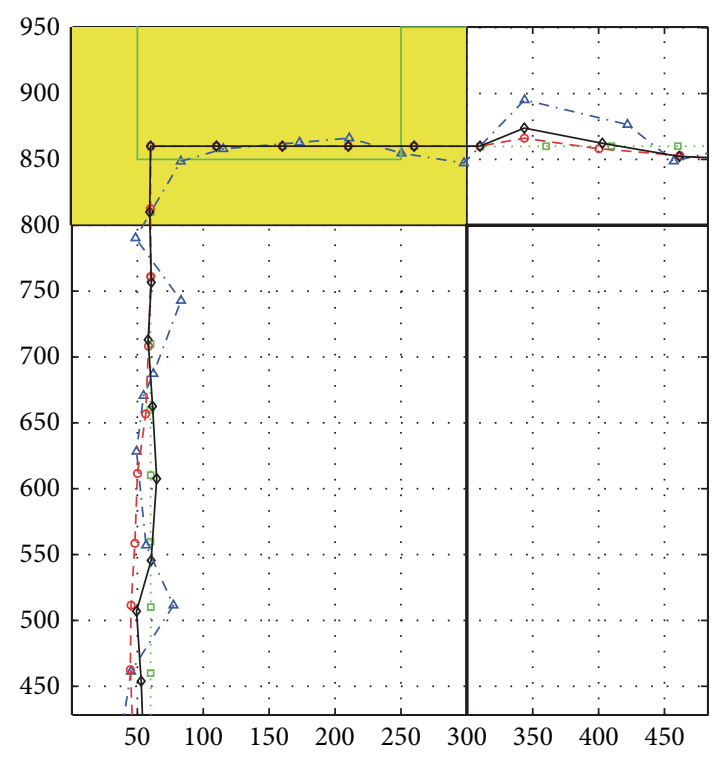

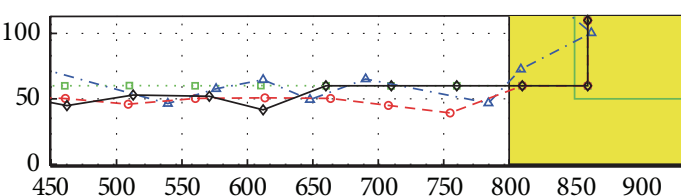

(b)

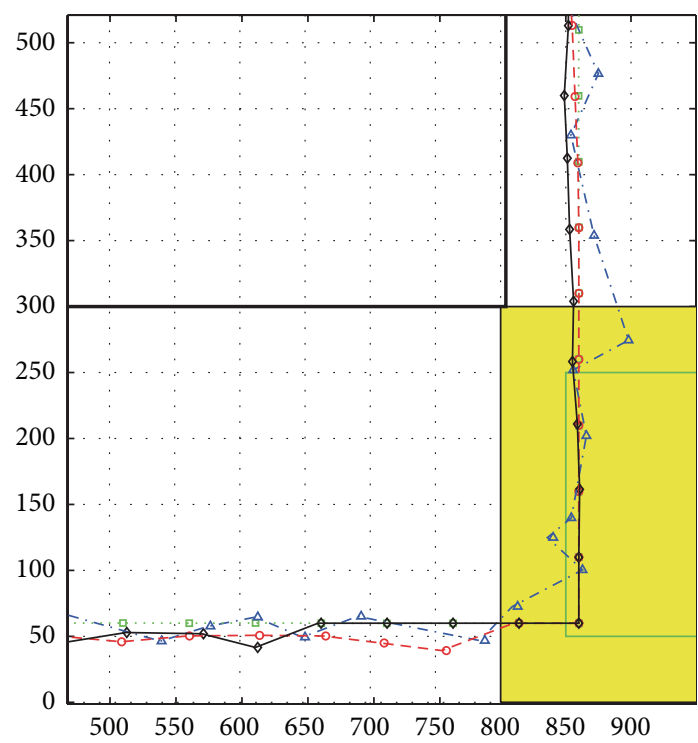

(c)

Figure 8: Magnification of one segment of the trajectories: (a) the first half of the trajectory; (b) the latter half of the trajectory; (c) the converging effect of the electronic tag floors.

TABLE 3: Comparison of the total errors of DR and the proposed method before and after formation correction.

\begin{tabular}{lcccccccc}
\hline$/ \mathrm{cm}$ & 1 & 2 & 3 & 4 & 5 & 6 & 7 & Mean values \\
\hline $\mathrm{DR}$ & 408.42 & 381.79 & 395.39 & 385.98 & 386.36 & 397.77 & 397.97 & 393.38 \\
$\mathrm{DR}_{\text {after }}$ & 377.97 & 351.67 & 362.07 & 354.06 & 355.66 & 365.00 & 369.08 & 362.22 \\
$\mathrm{PM}$ & 360.75 & 339.54 & 349.26 & 339.88 & 344.04 & 350.76 & 350.07 & 347.76 \\
$\mathrm{PM}_{\text {after }}$ & 335.28 & 310.53 & 392.92 & 307.89 & 194.80 & 362.34 & 265.90 & 309.95 \\
\hline
\end{tabular}

distributed tags. Figures 13 and 14 show the environment and the robots, respectively.

The robots move along the preset path inside the scope of 8 reference nodes while maintaining the desired formation of a square shape. Since they move along the four sides of a square and every robot is located in the vertices of the $4 \times 4$ square formation, each robot in the swarm will be assigned as the global leader, group leader, and follower during the whole movement course. They experience the same localization process and the behavior of each robot is equivalent. For the simplicity of discussion, the one located in $(4,4)$ of the formation is studied. Figure 15 is the estimated trajectory of the DR method and the DR method with RFID calibration compared with the real trajectory. During the process of moving along the first side of the $5 \times 5 \mathrm{~m}^{2}$ square, the estimated positions are relatively close to robots' real location. However, as the robots move further, the localization results are not satisfactory. The estimated trajectory of DR diverges farther and farther away from the real trajectory. Figure 16 shows the increasing deviation of localization using the DR method. However, there is a downward trend in the deviation of DR method between the 24th and the 35th sampling step in Figure 16. This phenomenon results from the accumulated errors as the robots take more turns along their path. It can be seen from Figure 15 that the estimated position of the robot is ahead of the real coordinates. Thus, with an inaccurate turning angle estimation towards the direction of the robot's actual trajectory, the localization results of DR method are more close to the real position than the proposed method. The real localization performances are shown by the average deviation values of the two localization methods in the far right of Figure 16.

As shown in Figure 17, after integrating the DR method with the WSN and electronic tag floors, the performance of localization system is greatly improved. The advantage of the proposed method is not obvious in the first few steps, but it gives much more precise estimated location of the robot swarm in the long term. The stability of the 


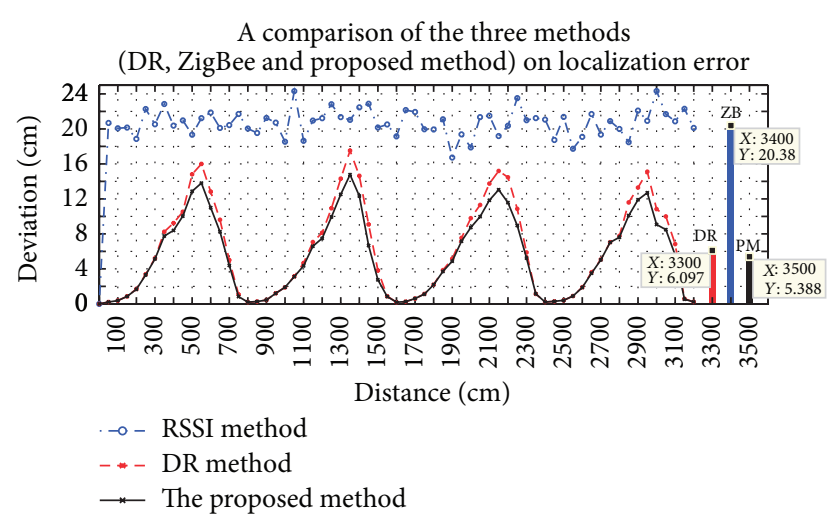

FIGURE 9: Localization errors of the leader robot using DR, ZigBee, and the proposed method.

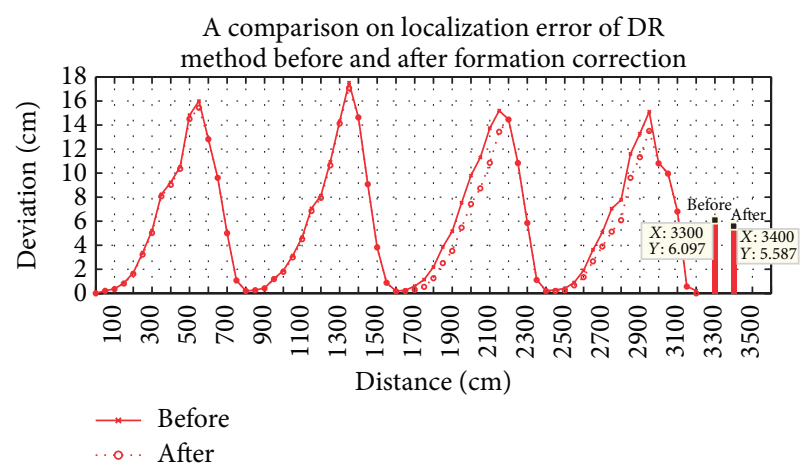

FIGURE 10: Comparison on localization errors of DR before and after formation correction.

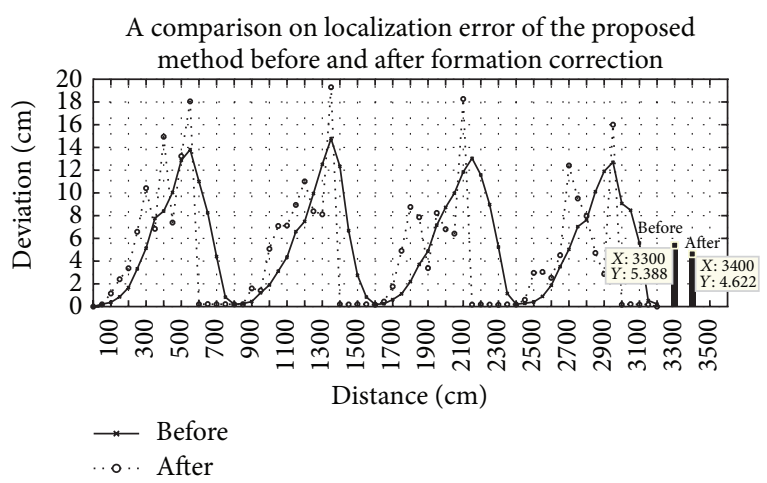

FIGURE 11: Comparison on localization error of the proposed method before and after formation correction.

proposed method is also superior to the DR method, which is illustrated by Figure 18. The deviation of proposed method is not accumulating and remains in a certain range. There is a significant drop of $11 \mathrm{~cm}$ in the mean localization error for each step.

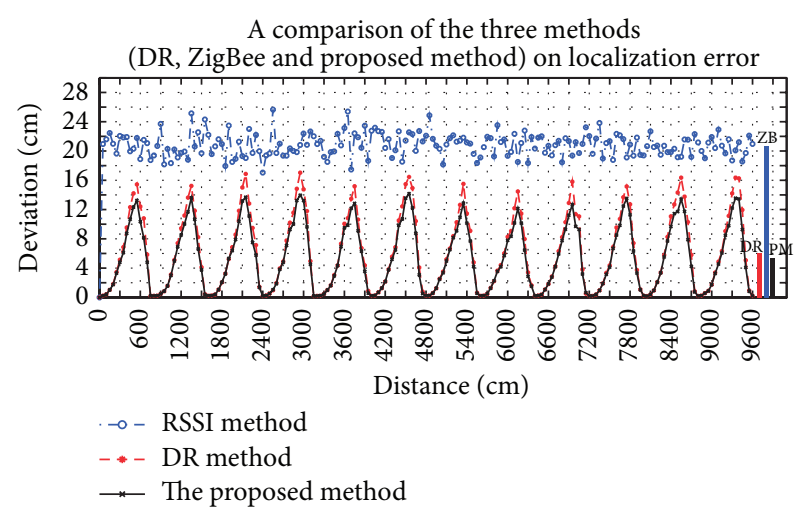

FIGURE 12: Localization errors of the leader robot using DR, ZigBee, and the proposed method during the movement of the first two rounds.

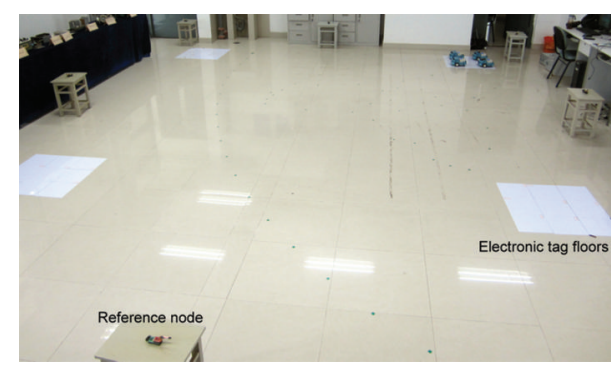

FIGURE 13: Experimental environment and the localization system.

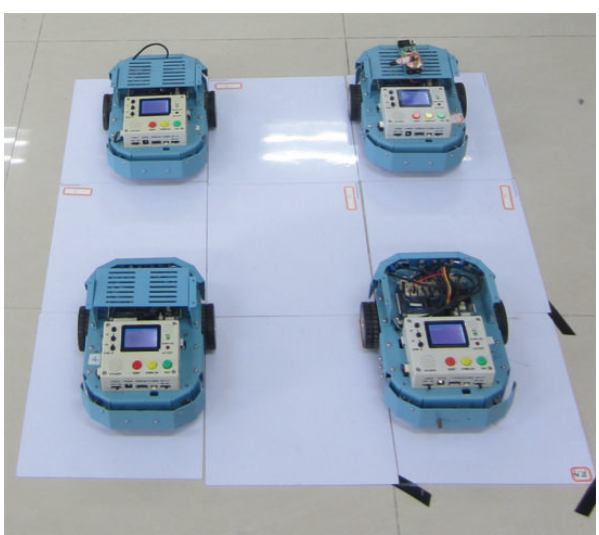

FIgURE 14: The robots used for the experiments.

\section{Conclusions}

Precise localization is the basis for the performance of a swarm robotic system. The dead reckoning-based localization method is easy to implement, yet it has unbounded accumulated errors and too much uncertainty due to stochastic errors. ZigBee-based WSN localization has less uncertainty and its error does not accumulate, but it has the disadvantage of low precision for short range of localization. The proposed method in this paper integrates the two methods with Kalman filter and uses the RFID technique to eliminate 


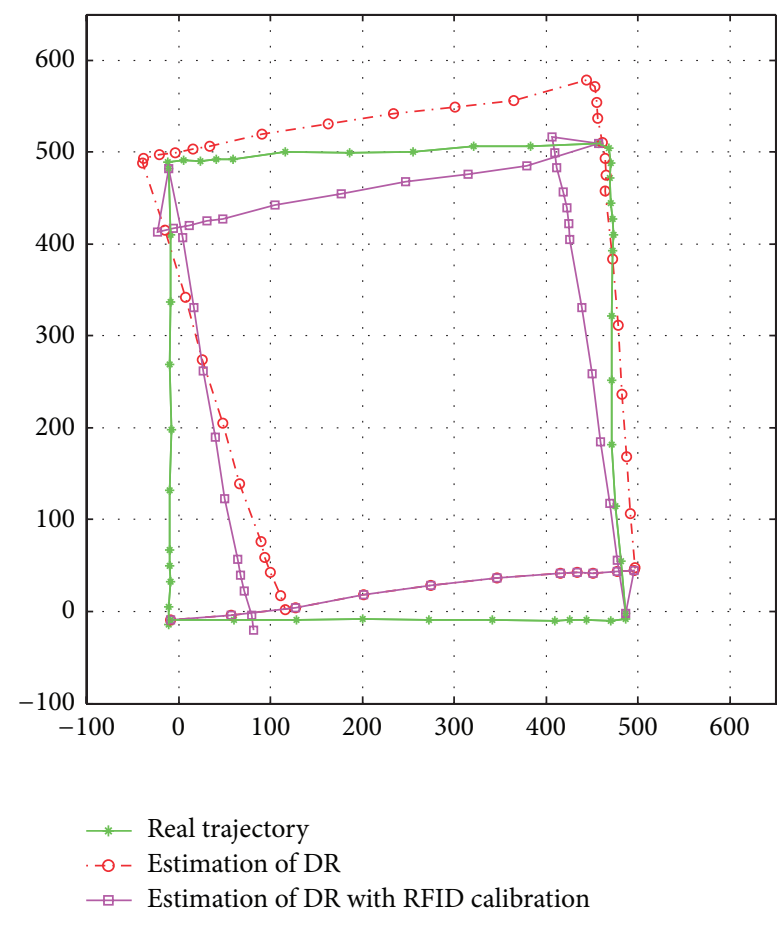

FIGURE 15: Performances of DR with and without RFID calibration.

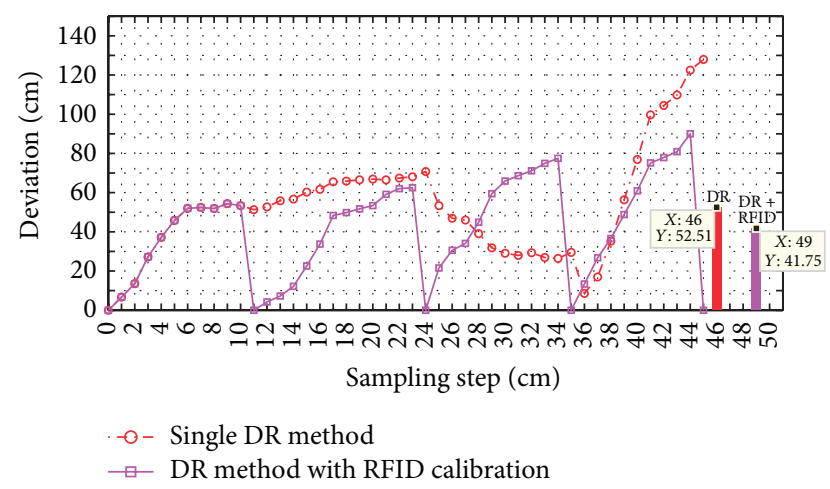

FIGURE 16: Deviation of the trajectories of the DR method.

the accumulated errors. Both of the simulation results and real experiments demonstrate the success of the proposed method. Our future work will focus on theoretical analysis of the swarm robotic systems with precise localization and learning-based methods $[31,32]$ for the coordinated control of swarm robots in an uncertain environment.

\section{Conflict of Interests}

The authors declare that there is no conflict of interests regarding the publication of this paper.

\section{Acknowledgment}

This work was supported by the Natural Science Foundation of China (nos. 61273327 and 61432008).

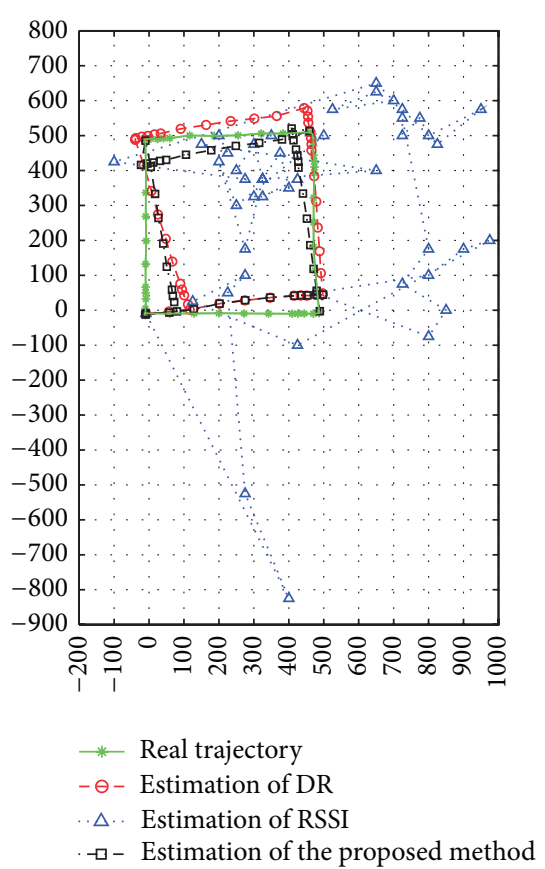

FIGURE 17: Real trajectory and the estimated trajectories of DR, RSSI, and the proposed method.

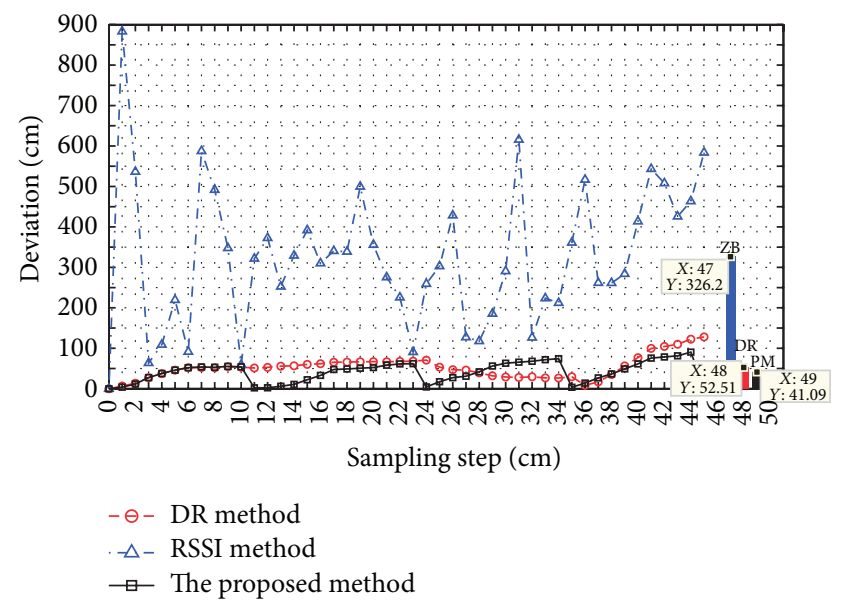

FIGURE 18: Deviations of the trajectories of DR, RSSI, and the proposed method.

\section{References}

[1] G. Beni, "From swarm intelligence to swarm robotics," in Swarm Robotics, pp. 1-9, Springer, Berlin, Germany, 2005.

[2] S. Qu, C. Chen, and D. Dong, "Behavior-based navigation control of swarm robots with improved potential field," in Proceedings of the 33rd Chinese Control Conference, pp. 84628467, Nanjing, China, July 2014.

[3] E. Şahin, "Swarm robotics: from sources of inspiration to domains of application," in Swarm Robotics, Springer, Berlin, Germany, 2005.

[4] T. Fukuda, Y. Kawauchi, and F. Hara, "A study on a dynamically reconfigurable robotic system. (Self-organizing distributed 
intelligence system of learning and reasoning for cellular robotics "CEBOT")," JSME International Journal, Series C: Dynamics, Control, Robotics, Design and Manufacturing, vol. 37, no. 1, pp. 162-171, 1994.

[5] R. Alami, F. Robert, F. Ingrad, and S. Suzuki, "Multi-robot cooperation through incremental plan-merging," in Proceedings of the IEEE International Conference on Robotics and Automation, pp. 2573-2579, IEEE, May 1995.

[6] M. Dorigo, E. Tuci, F. Mondada et al., "The swarm-bots project," in Swarm Robotics, pp. 31-44, Springer, Berlin, Germany, 2005.

[7] J. Seyfried, M. Szymanski, N. Bender, R. Estaña, M. Thiel, and H. Wörn, "The I -SWARM project: intelligent small world autonomous robots for micro-manipulation," in Swarm Robotics, vol. 3342 of 83Lecture Notes in Computer Science, pp. 70-83, Springer, Berlin, Germany, 2005.

[8] J. H. Kim and H. S. Cho, "An improved dead reckoning scheme for a mobile robot using neutral networks," Mechatronics, vol. 3, no. 5, pp. 625-645, 1993.

[9] M. Hashimoto, F. Oba, K. Imamaki, and T. Nishida, "Mobile robot localization using integrated dead reckoning and laser/ corner cube based location systems," in Robotics, Mechatronics and Manufacturing Systems, pp. 403-408, Elsevier, 1993.

[10] G. Mao, B. Fidan, and B. D. O. Anderson, "Wireless sensor network localization techniques," Computer Networks, vol. 51, no. 10, pp. 2529-2553, 2007.

[11] T. Ishimoto and S. Hara, "Use of RSSI for motion control of wirelessly networked robot swarm," in Proceedings of the International Workshop on Robotic and Sensors Environments, pp. 92-97, IEEE, 2008.

[12] L. Oliveira, H. Li, L. Almeida, and T. E. Abrudan, "RSSI-based relative localisation for mobile robots," Ad Hoc Networks, vol. 13, pp. 321-335, 2014.

[13] N. Patwari, A. O. Hero, M. Perkins, N. S. Correal, and R. J. O'Dea, "Relative location estimation in wireless sensor networks," IEEE Transactions on Signal Processing, vol. 51, no. 8, pp. 2137-2148, 2003.

[14] N. Patwari, J. N. Ash, S. Kyperountas, A. O. Hero III, R. L. Moses, and N. S. Correal, "Locating the nodes: cooperative localization in wireless sensor networks," IEEE Signal Processing Magazine, vol. 22, no. 4, pp. 54-69, 2005.

[15] R.-B. Zhang, J.-G. Guo, F.-H. Chu, and Y.-C. Zhang, "Environmental-adaptive indoor radio path loss model for wireless sensor networks localization," International Journal of Electronics and Communications, vol. 65, no. 12, pp. 1023-1031, 2011.

[16] H. S. Dulimarta and A. K. Jain, "Mobile robot localization in indoor environment," Pattern Recognition, vol. 30, no. 1, pp. 99111, 1997.

[17] T. Balch and R. C. Arkin, "Behavior-based formation control for multirobot teams," IEEE Transactions on Robotics and Automation, vol. 14, no. 6, pp. 926-939, 1998.

[18] H. Takahashi, H. Nishi, and K. Ohnishi, "Autonomous decentralized control for formation of multiple mobile robots considering ability of robot," IEEE Transactions on Industrial Electronics, vol. 51, no. 6, pp. 1272-1279, 2004.

[19] Y. Chen and Z. Wang, "Formation control: a review and a new consideration," in Proceedings of the IEEE IRS/RSJ International Conference on Intelligent Robots and Systems (IROS '05), pp. 3181-3186, August 2005.

[20] R. Haghighi and C. C. Cheah, "Multi-group coordination control for robot swarms," Automatica, vol. 48, no. 10, pp. 25262534, 2012.
[21] G. Zhou, T. He, S. Krishnamurthy, and J. A. Stankovic, "Models and solutions for radio irregularity in wireless sensor networks," ACM Transactions on Sensor Networks, vol. 2, no. 2, pp. 221-262, 2006.

[22] H. F. Durrant-Whyte, B. Y. S. Rao, and H. Hu, "Toward a fully decentralized architecture for multi-sensor data fusion," in Proceedings of the IEEE International Conference on Robotics and Automation, vol. 2, pp. 1331-1336, Cincinnati, Ohio, USA, May 1990.

[23] J. J. Leonard and H. F. Durrant-Whyte, "Mobile robot localization by tracking geometric beacons," IEEE Transactions on Robotics and Automation, vol. 7, no. 3, pp. 376-382, 1991.

[24] L. Jetto, S. Longhi, and G. Venturini, "Development and experimental validation of an adaptive extended Kalman filter for the localization of mobile robots," IEEE Transactions on Robotics and Automation, vol. 15, no. 2, pp. 219-229, 1999.

[25] S. Kwon, K. Yang, and S. Park, "An effective kalman filter localization method for mobile robots," in Proceedings of the IEEE/RSJ International Conference on Intelligent Robots and Systems, pp. 1524-1529, October 2006.

[26] G. Welch and G. Bishop, "An introduction to the Kalman filter," Tech. Rep. 95-04, University of North Carolina, 1995.

[27] Q. Gan and C. J. Harris, "Comparison of two measurement fusion methods for Kalman-filter-based multisensor data fusion," IEEE Transactions on Aerospace and Electronic Systems, vol. 37, no. 1, pp. 273-279, 2001.

[28] G. Fu, J. Zhang, W. Chen, F. Peng, P. Yang, and C. Chen, "Precise localization of mobile robots via odometry and wireless sensor network," International Journal of Advanced Robotic Systems, vol. 10, article no. 203, 2013.

[29] C. M. Roberts, "Radio frequency identification (RFID)," Computers and Security, vol. 25, no. 1, pp. 18-26, 2006.

[30] X. Zhu, S. K. Mukhopadhyay, and H. Kurata, "A review of RFID technology and its managerial applications in different industries," Journal of Engineering and Technology Management, vol. 29, no. 1, pp. 152-167, 2012.

[31] C. Chen, D. Dong, H.-X. Li, J. Chu, and T.-J. Tarn, "Fidelitybased probabilistic Q-learning for control of quantum systems," IEEE Transactions on Neural Networks and Learning Systems, vol. 25, no. 5, pp. 920-933, 2014.

[32] D. Dong, C. Chen, H. Li, and T.-J. Tarn, "Quantum reinforcement learning," IEEE Transactions on Systems, Man, and Cybernetics Part B: Cybernetics, vol. 38, no. 5, pp. 1207-1220, 2008. 


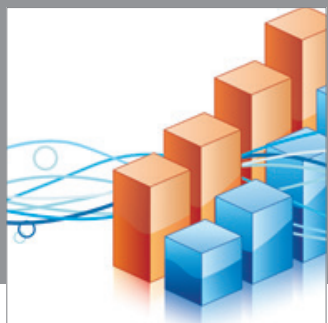

Advances in

Operations Research

mansans

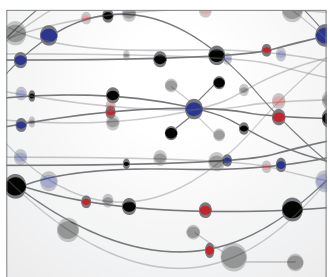

The Scientific World Journal
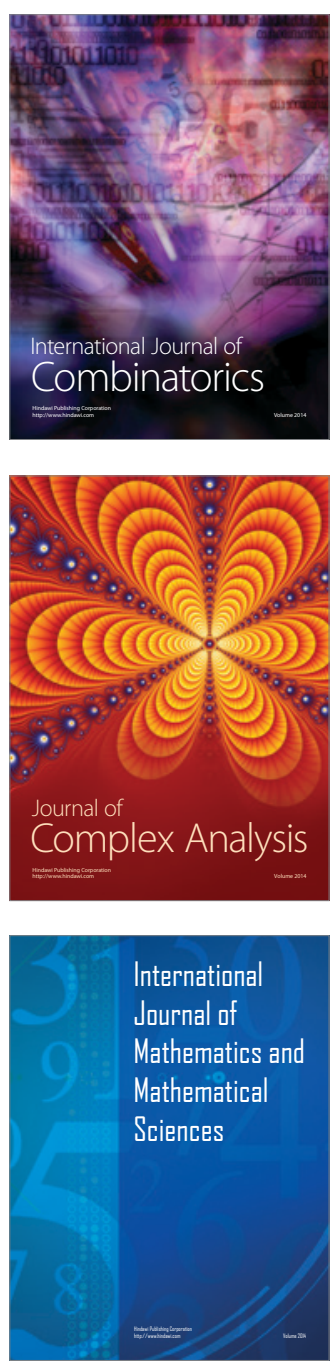
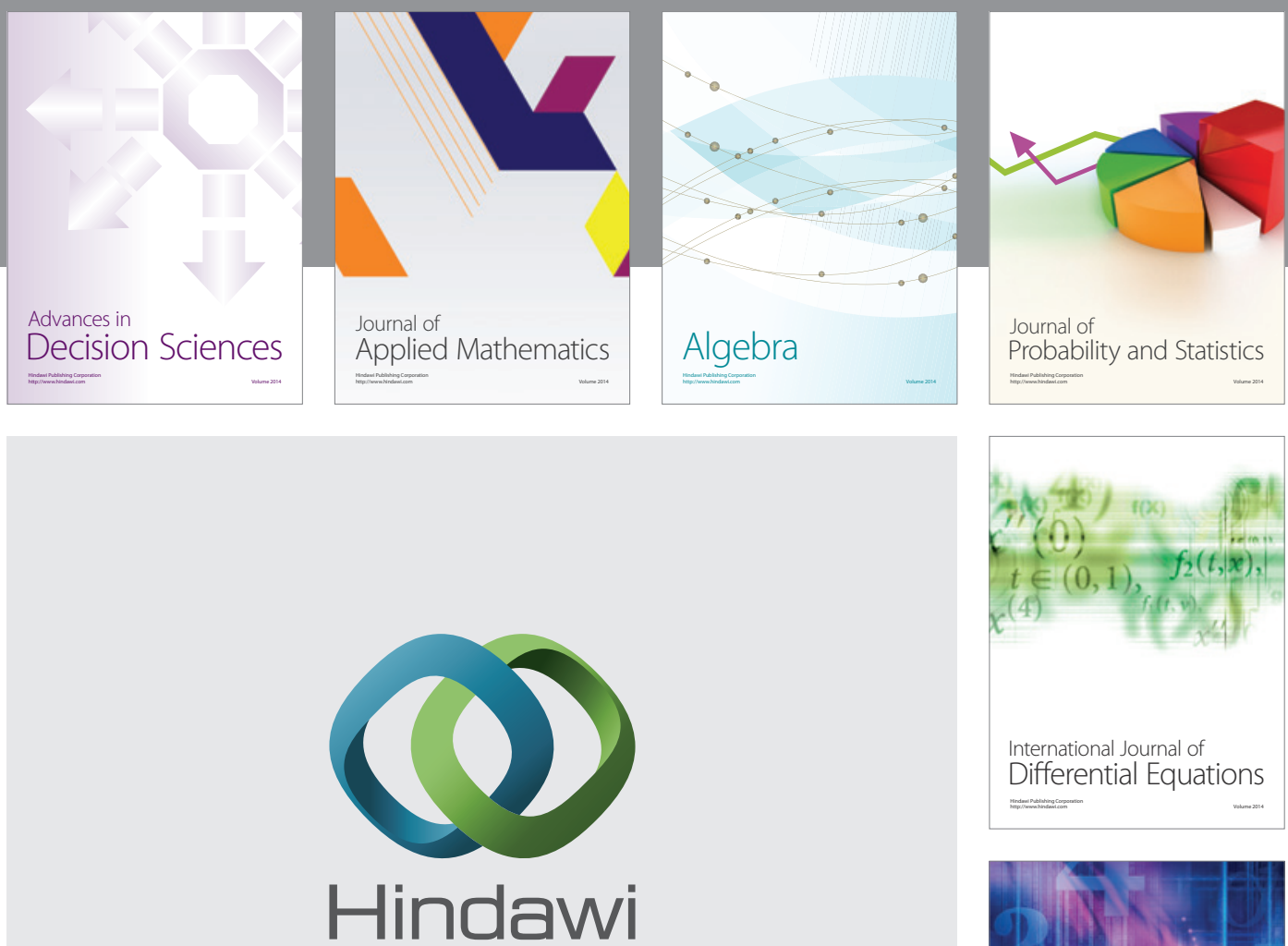

Submit your manuscripts at http://www.hindawi.com
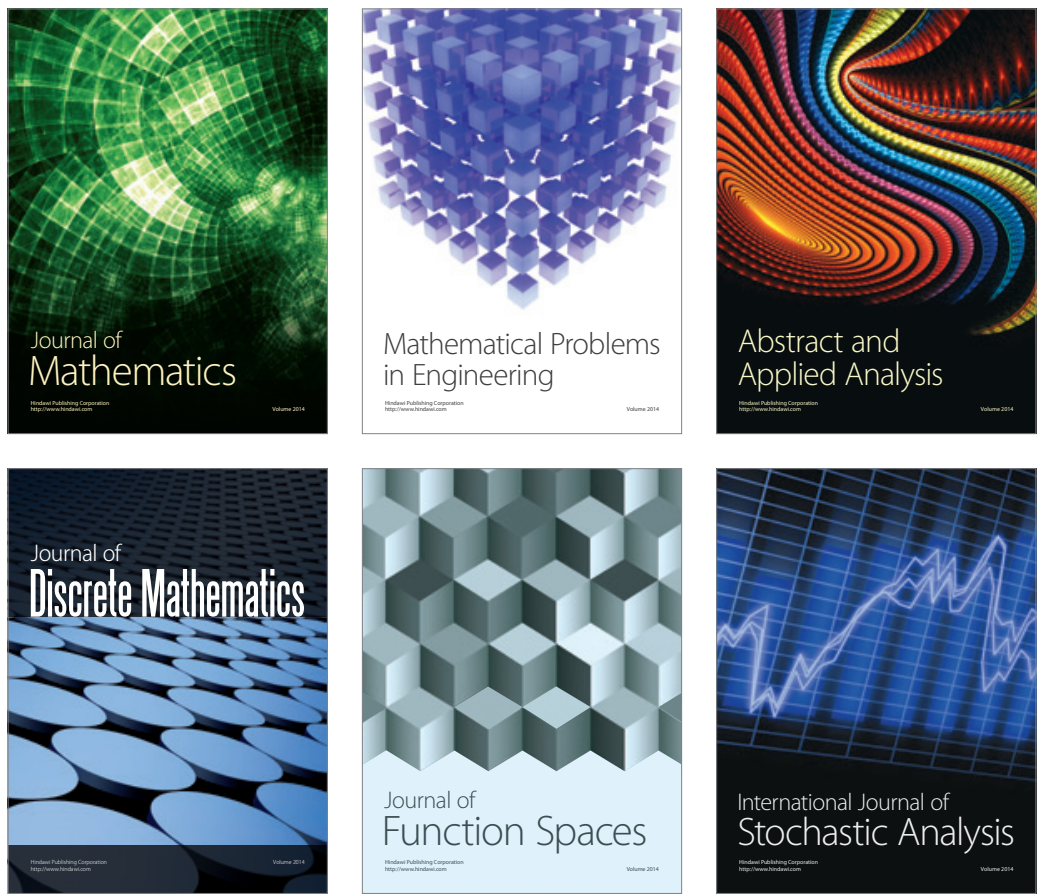

Journal of

Function Spaces

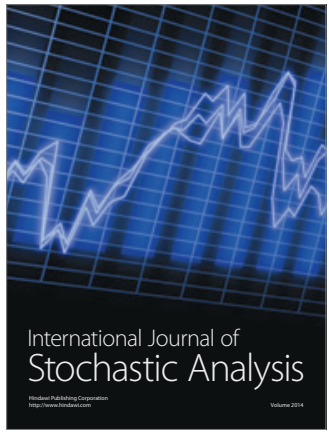

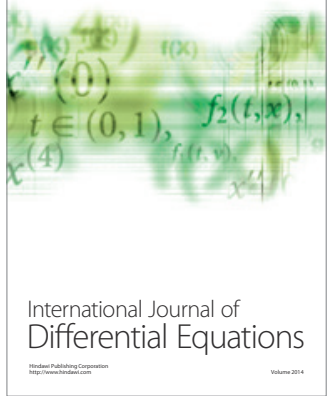
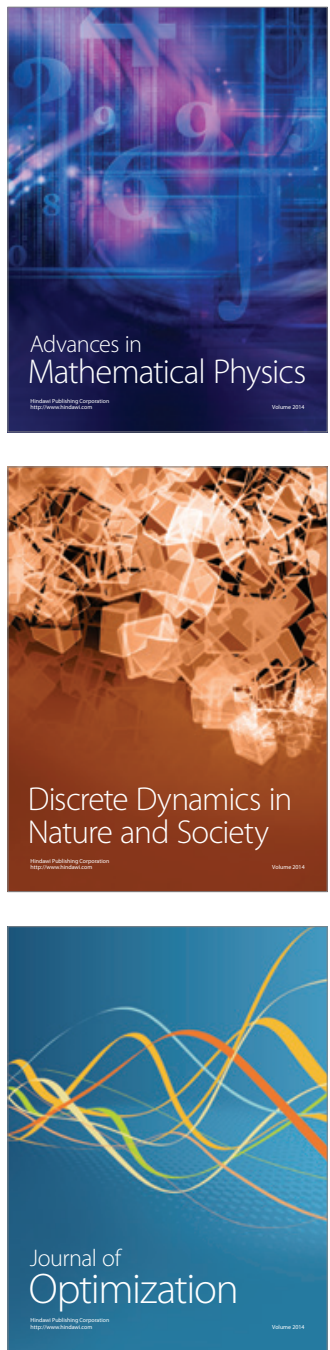